\title{
Molecular dynamics ensemble refinement of the heterogeneous native state of NCBD using chemical shifts and NOES
}

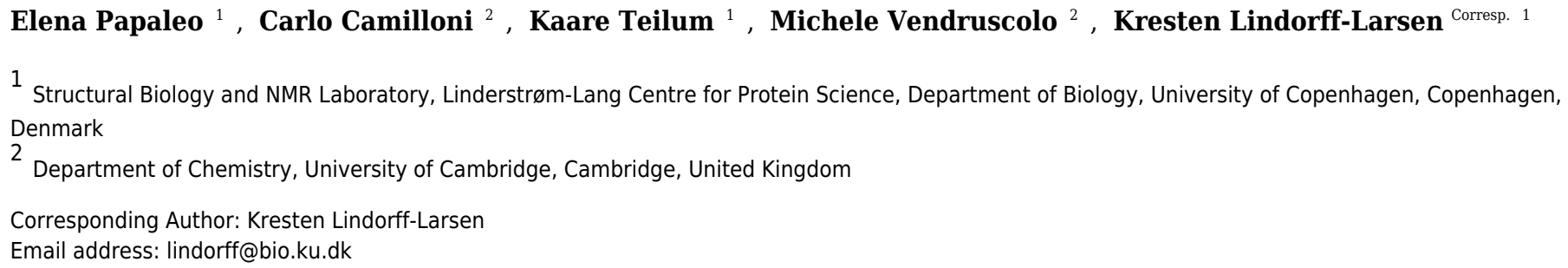

Many proteins display complex dynamical properties that are often intimately linked to their biological functions. As the native state of a protein is best described as an ensemble of conformations, it is important to be able to generate models of native state ensembles with high accuracy. Due to limitations in sampling efficiency and force field accuracy it is, however, challenging to obtain accurate ensembles of protein conformations by the use of molecular simulations alone. Here we show that dynamic ensemble refinement, which combines an accurate atomistic force field with commonly available nuclear magnetic resonance (NMR) chemical shifts and NOEs, can provide a detailed and accurate description of the conformational ensemble of the native state of a highly dynamic protein. As both NOEs and chemical shifts are averaged on timescales up to milliseconds, the resulting ensembles reflect the structural heterogeneity that goes beyond that probed e.g. by NMR relaxation order parameters. We selected the small protein domain NCBD as object of our study since this protein, which has been characterized experimentally in substantial detail, displays a rich and complex dynamical behaviour. In particular, the protein has been described as having a molten-globule like structure, but with a relatively rigid core. Our approach allowed us to describe the conformational dynamics of NCBD in solution, and to probe the structural heterogeneity resulting from both short- and longtimescale dynamics by the calculation of order parameters on different time scales. These results illustrate the usefulness of our approach since they show that NCBD is rather rigid on the nanosecond timescale, but interconverts within a broader ensemble on longer timescales, thus enabling the derivation of a coherent set of conclusions from various NMR experiments on this protein, which could otherwise appear in contradiction with each other. 


\section{Molecular dynamics ensemble refinement of the heterogeneous 2 native state of NCBD using chemical shifts and NOEs}

3 Elena Papaleo ${ }^{1,2}$, Carlo Camilloni ${ }^{3,4}$, Kaare Teilum ${ }^{1}$, Michele Vendruscolo ${ }^{3}$ and Kresten Lindorff4 Larsen $^{1}$

$5{ }^{1}$ Structural Biology and NMR Laboratory, Linderstrøm-Lang Centre for Protein Science, Department of Biology, 6 University of Copenhagen, Ole Maaløes Vej 5, DK-2200 Copenhagen, Denmark

$7 \quad{ }^{3}$ Department of Chemistry, University of Cambridge, Cambridge CB2 1EW, United Kingdom

10 Corresponding Author

11 Kresten Lindorff-Larsen

12 Email address: lindorff@bio.ku.dk

Present Addresses

$15{ }^{2}$ Computational Biology Laboratory, Danish Cancer Society Research Center, Copenhagen, 16 Denmark

$17{ }^{4}$ Department of Biosciences, University of Milano, Milano, Italy 


\section{ABSTRACT}

Many proteins display complex dynamical properties that are often intimately linked to their biological functions. As the native state of a protein is best described as an ensemble of conformations, it is important to be able to generate models of native state ensembles with high accuracy. Due to limitations in sampling efficiency and force field accuracy it is, however, challenging to obtain accurate ensembles of protein conformations by the use of molecular simulations alone. Here we show that dynamic ensemble refinement, which combines an accurate atomistic force field with commonly available nuclear magnetic resonance (NMR) chemical shifts and NOEs, can provide a detailed and accurate description of the conformational ensemble of the native state of a highly dynamic protein. As both NOEs and chemical shifts are averaged on timescales up to milliseconds, the resulting ensembles reflect the structural heterogeneity that goes beyond that probed e.g. by NMR relaxation order parameters. We selected the small protein domain NCBD as object of our study since this protein, which has been characterized experimentally in substantial detail, displays a rich and complex dynamical behaviour. In particular, the protein has been described as having a molten-globule like structure, but with a relatively rigid core. Our approach allowed us to describe the conformational dynamics of NCBD in solution, and to probe the structural heterogeneity resulting from both short- and long-timescale dynamics by the calculation of order parameters on different time scales. These results illustrate the usefulness of our approach since they show that NCBD is rather rigid on the nanosecond timescale, but interconverts within a broader ensemble on longer timescales, thus enabling the derivation of a coherent set of conclusions from various NMR experiments on this protein, which could otherwise appear in contradiction with each other.

\section{INTRODUCTION}

Molecular dynamics (MD) simulations have the potential ability to provide an accurate, atomiclevel description of the conformational ensembles of proteins and their macromolecular complexes (Lindorff-Larsen et al., 2005; Dror et al., 2012; Perilla et al., 2015). Nevertheless, simulations are limited by both the accuracy of the physical models (force fields) and the precision due to conformational sampling (Mobley, 2012; Esteban-Martín, Fenwick \& Salvatella, 2012). To overcome these problems, it is possible to bias the simulations using experimental data as structural restraints taking into account the inherent averaging in the experiments (Lindorff-Larsen et al., 
52 2005; Camilloni et al., 2012; Lehtivarjo et al., 2012; Pitera \& Chodera, 2012; Camilloni \&

53 Vendruscolo, 2014; Ravera et al., 2016). In this way, the experimental data can be included as a 54 system-specific force-field correction, that combines the two sources of information using 55 Bayesian statistics or the maximum entropy principle (Pitera \& Chodera, 2012; Roux \& Weare, 56 2013; Cavalli, Camilloni \& Vendruscolo, 2013; Boomsma, Ferkinghoff-Borg \& Lindorff-Larsen, 2014; White \& Voth, 2014; Olsson et al., 2014; MacCallum, Perez \& Dill, 2015; Hummer \& Köfinger, 2015; Bonomi et al., 2016, 2017; Bottaro et al., 2018). Among the many techniques that can be used to probe structure and dynamics of proteins, NMR spectroscopy stands out as being able to provide a number of different parameters that are sensitive to protein dynamics over different timescales, as well as to probe the "average structure" in solution.

Previously, replica-averaged simulations have provided a wealth of information about the dynamical ensembles that proteins can attain in solution (Lindorff-Larsen et al., 2005; Tang, Schwieters \& Clore, 2007; Fenwick et al., 2011; Camilloni et al., 2012; Ángyán \& Gáspári, 2013; Camilloni, Cavalli \& Vendruscolo, 2013a,b; Islam et al., 2013; Vögeli et al., 2014; Camilloni \& Vendruscolo, 2014). Exploiting improvements in the accuracy and speed of predicting protein NMR chemical shifts from protein structure, (Kohlhoff et al., 2009; Han et al., 2011; Li \& Brüschweiler, 2012) it is now possible to combine experimental chemical shifts with molecular simulations to study protein structure and dynamics (Wishart \& Case, 2001; Cavalli et al., 2007; Shen et al., 2008; Wishart et al., 2008; Robustelli et al., 2009, 2010; Boomsma et al., 2014). In particular, chemical shifts can be used as replica-averaged structural restraints to determine the conformational fluctuations in proteins (Camilloni et al., 2012; Camilloni, Cavalli \& Vendruscolo, 2013a,b; Kannan et al., 2014; Kukic et al., 2014; Krieger et al., 2014). By using experimental data as a "system specific force field correction" (Boomsma, Ferkinghoff-Borg \& Lindorff-Larsen, 2014) such experimentally-restrained simulations remove some of the uncertainty associated with imperfect force fields and sampling (Tiberti et al., 2015; Löhr, Jussupow \& Camilloni, 2017).

Previously, we developed a dynamic-ensemble refinement (DER) approach for determining simultaneously the structure and dynamics of proteins by combining distance restraints from nuclear Overhauser effect (NOE) experiments, dynamical information from relaxation order parameters and MD simulations (Lindorff-Larsen et al., 2005). Similarly, it has been demonstrated that accurate ensembles of conformations that represent longer timescale dynamics can be obtained 
83 applications have, however, relied on a type of data (relaxation order parameters or residual dipolar 84 couplings) that may not be readily available.

85 We therefore sought to extend this approach to study conformational variability using more 86 commonly available data, thus making the DER method more generally applicable. We thus focus on using NMR chemical shifts and NOEs as these are both commonly available and are averaged over long, millisecond timescales. We demonstrate the potential by describing the structural heterogeneity of a highly dynamic protein. Our method relies on supplementing the sparse experimental data with the experimentally-validated CHARMM22* force field (Piana, LindorffLarsen \& Shaw, 2011), which provides a relatively accurate description of the subtle balance among the stability of the different secondary structure classes, and which has been shown to provide a good description of many structural and dynamical aspects related to protein structure (Shaw et al., 2010; Lindorff-Larsen et al., 2012a,b; Piana, Lindorff-Larsen \& Shaw, 2012; Papaleo et al., 2014; Rauscher et al., 2015). Our hypothesis was that using a more accurate force field would make it possible to determine an accurate ensemble from less information-rich experimental data. In particular, though chemical shifts in principle contain very detailed information, this information is difficult to extract using current methods.

As object of our study we selected NCBD (the Nuclear Coactivator Binding Domain) of CBP (CREB Binding Protein), a 59-residue protein domain that has been experimentally characterized in substantial detail. Experiments on NCBD have revealed a rich and complex dynamical behaviour of the protein in solution (Demarest et al., 2004; Ebert et al., 2008; Kjaergaard, Teilum \& Poulsen, 2010; Kjaergaard, Poulsen \& Teilum, 2012; Kjaergaard et al., 2013). For a protein of its size, NCBD displays surprisingly broad NMR peaks, suggestive of conformational heterogeneity with relatively slow interconversion between different states. Nevertheless, it was possible to assign both backbone and side chain chemical shifts and determine a number of conformationally-averaged inter-nuclear distances, including a few long-range contacts, via NOE experiments (Ebert et al., 2008; Kjaergaard, Teilum \& Poulsen, 2010; Kjaergaard, Poulsen \& Teilum, 2012). NMR relaxation experiments suggest that the protein, at least on the nanosecond

110 timescale, is relatively rigid (Kjaergaard, Poulsen \& Teilum, 2012). NCBD forms complexes with 111 several other proteins, where it intriguingly folds into remarkably different tertiary structures

112 (Demarest et al., 2002; Qin et al., 2005). For example, the structure of NCBD in complex with 113 ACTR (Demarest et al., 2002) and certain other partners (Waters et al., 2006; Lee et al., 2010) 
114 resembles the average structure populated by NCBD in the absence of binding partners (Figure 1),

115 whereas the structure of NCBD is markedly different when bound to the protein IRF-3 (Qin et al.,

116 2005). Thus, the dynamical properties of NCBD, and its ability to adopt different conformations,

117 appear crucial for its diverse biological functions.

118 Our results show that a dynamic ensemble refinement that combines NOEs, chemical shifts and

119 the CHARMM22* force field provides a rather accurate description of the structural dynamics of

120 the ground state structure of NCBD. We show via cross-validation with independent NMR data

121 that all three components (the two sources of experimental information and the force field)

122 contribute to the overall accuracy. The ensemble that we obtained reveals a relatively broad

123 distribution of conformations, reflecting the conformational heterogeneity of NCBD on the

124 millisecond timescale. Further, we quantified the level of structural fluctuations that would be

125 measured by relaxation experiments and demonstrate that, on the nanosecond timescale, NCBD is

126 more rigid, thus helping to reconcile earlier conflicting views of this protein.

127

\section{MATERIALS AND METHODS}

129 Ensemble generation. MD simulations were performed using Gromacs 4.5, (Pronk et al., 2013)

130 coupled to a modified version of Plumed 1.3, (Bonomi et al., 2009) and using either the 131 CHARMM22* (Piana, Lindorff-Larsen \& Shaw, 2011) or CHARMM22 (MacKerell, et al., 1998)

132 force fields. As starting structure for most simulations we used the first conformer from a 133 previously determined NMR structure of free NCBD as deposited in the PDB entry 2KKJ 134 (Kjaergaard, Teilum \& Poulsen, 2010). To evaluate the effect of our choice of the initial structure, 135 we also performed one simulation starting from an alternative NCBD conformation (PDB entry: 136 1ZOQ, chain C) (Qin et al., 2005). Missing residues in 1ZOQ (compared to 2KKJ) were rebuilt 137 by Modeller 9.11 (Fiser \& Šali, 2003).

138 The protein was embedded in a dodecahedral box containing 8372 TIP3P water molecules 139 (Jorgensen et al., 1983) and simulated using periodic boundary conditions with a 2 fs timestep and 140 LINCS constraints (Hess et al., 1993). Production simulations were performed in the NVT 141 ensemble with the Bussi thermostat (Bussi, Donadio \& Parrinello, 2007) using a pre-equilibrated 142 starting structure for which the volume was selected based on a short NPT simulation. $\mathrm{NaCl}$ was 143 added to a concentration of $\sim 20 \mathrm{mM}$ to reproduce the experimental conditions at which chemical 
144 shifts and NOEs were determined (Kjaergaard, Teilum \& Poulsen, 2010). The van der Waals and

145 short-range electrostatic interactions were truncated at $9 \AA$, whereas long-range electrostatic

146 effects were treated with the particle mesh Ewald method (Essmann et al., 1995).

147 We carried out MD simulations with replica-averaged experimental restraints using 1, 2, 4 or 8 148 replicas (Table S1 gives an overview of the simulations that were performed). The use of replica149 averaged restrained simulations enables us to use different equilibrium experimental observable 150 as a restraint in MD simulation in a way that minimises the risk of over restraining because replica151 averaging is a practical implementation of the maximum entropy principle. As a control we also 152 performed a simulation that was not biased by any experimental restraints (i.e. an unbiased 153 simulation). To examine the role played by each of the different types of experimental data, we 154 also performed simulations in which we included different combinations of the experimental 155 restraints: chemical shifts only (CS), NOEs only (NOE), and both chemical shifts and NOEs (CS$156 \mathrm{NOE}$ ). In the simulations, each replica was evolved through a series of simulated annealing (SA) 157 cycles between 304 and $454 \mathrm{~K}$ for a total duration of $0.6 \mathrm{~ns}$ per cycle. Specifically, for each SA 158 cycle we performed: (i) 100 ps at $304 \mathrm{~K}$, (ii) a linear increase of the temperature from 304 to $454 \mathrm{~K}$ 159 over 100 ps, (iii) 100 ps at 454K, and (iv) a linear cooling from $454 \mathrm{~K}$ to $304 \mathrm{~K}$ in the remaining 160300 ps. Each new cycle was initiated from the final structure from the previous cycle. We only 161 used structures from the $304 \mathrm{~K}$ portions of the simulations for our analyses, corresponding also to 162 the temperature at which the NMR data were recorded (Kjaergaard, Teilum \& Poulsen, 2010). 163 Example scripts for performing the simulations are available as supporting information.

164 Chemical shifts for the backbone atoms (C $\alpha, C^{\prime}, \mathrm{H} \alpha, \mathrm{H}$ and $\mathrm{N}$ ) and $\mathrm{C} \beta \mathrm{CS}$ (deposited in BMRB 165 entry 16363) were used as restraints (with the exception of the C $\beta$ of glutamines, which we have 166 sometimes found to be imprecisely predicted). The resulting dataset includes $54 \mathrm{C} \alpha, 37 \mathrm{C} \beta, 52$ $167 \mathrm{H} \alpha$ and 48 C', H and $\mathrm{N}$ chemical shifts, respectively. The backbone chemical shifts cover most of 168 the NCBD sequence with the exception of the first four to six N-terminal residues, depending on 169 type of chemical shifts. The $\mathrm{C} \beta$ chemical shifts for the first seven N-terminal and last five C170 terminal residues, as well as for some residues of the loops connecting the $\alpha$-helices, are missing 171 with few exceptions.

172 During the structure determination protocol, chemical shifts were calculated by CamShift 173 (Kohlhoff et al., 2009) for all the nuclei for which an experimental value is available and then 
174 averaged over the replicas. The resulting average over the replicas was compared with the 175 experimental value, and the ensemble as a whole restrained using a harmonic function with a force 176 constant of $5.2 \mathrm{~kJ} \mathrm{~mol}^{-1} \mathrm{ppm}^{-2}$ (Camilloni et al., 2012; Camilloni, Cavalli \& Vendruscolo, 2013a).

177 At the higher temperatures, $T$, explored during the simulated annealing, the force constant was 178 scaled by a factor of $(304 \mathrm{~K} / T)$. The value of the force constant was chosen roughly to match the 179 calculated chemical shifts to experiments within the uncertainty of the CamShift predictor; the 180 experimental uncertainty of the chemical shifts is negligible in comparison.

181 NOE restraints were obtained by 455 NOE-derived distance intervals (Kjaergaard, Teilum \& 182 Poulsen, 2010) (BMRB entry 16363) of which 46 were long-range (i.e. separated by more than 4 183 residues). The proton-proton distances, $r$, were calculated and averaged as $r^{-6}$ over the replicas 184 (Tropp, 1980; Lindorff-Larsen et al., 2005). We used a flat-bottomed harmonic function 185 implemented in Gromacs to restrain the calculated averaged distances within the experimentally186 derived intervals. We used a variable force constant for the NOE-restraints during the SA cycles, 187 allowing the protein to sample more diverse structures in the high-temperature regime and thus to 188 decrease the risk of getting trapped in local minima. Force constants of 1000, 20 and $125 \mathrm{~kJ} \mathrm{~mol}^{-1}$ $189 \mathrm{~nm}^{-2}$ were used for the $304 \mathrm{~K}$ phase, a heating phase (from $304 \mathrm{~K}$ to $454 \mathrm{~K}$ ) and cooling phase (from $454 \mathrm{~K}$ to $304 \mathrm{~K})$, respectively.

191 In short, in the replica-averaged simulations we calculated at each step and for each replicaconformation the atomic distances that were measured by the NOE experiments and the backbone chemical shifts. These calculated single-conformer values were then averaged (linearly for the shifts and using $r^{-6}$ averaging for the distances) to determine the replica-averaged values, which were then compared to the experimentally determined values. Thus, the simulations penalize

196 deviations between the calculated ensemble averages and experimental values but allow 197 fluctuations of individual structures. In this way, the simulations are biased so as to agree with the experimental data as a whole, while allowing individual conformations to take on conformations whose NMR parameters differ from the experimentally derived averages.

200 To examine the role of the force field used in our approach, we compared the results from two 201 different force fields belonging to the same family (CHARMM). These force fields mostly differ 202 for the main-chain dihedral angle potential, as well a few parameters for certain side chains. 203 Specifically, we used either the CHARMM22* (Piana, Lindorff-Larsen \& Shaw, 2011) or 204 CHARMM22 (MacKerell, et al., 1998) force fields. The CHARMM22* force field is a refined 
205

206

207

208

209

210

211

212

213

214

215

216

217

218

219

220

221

222

223

224

225

226

227

228

229

230

231

232

233

234

235

version of CHARMM22 that includes modified backbone torsion angles optimized to give improved agreement with a range of NMR data in simulations of peptides of various lengths and secondary structure propensities. Further, in a previous comprehensive evaluation of protein force fields it, was demonstrated that these two force fields resulted in very different levels of agreement between simulations and experiments (Lindorff-Larsen et al., 2012a), making it possible for us to evaluate the importance of force field accuracy in restrained simulations.

Unbiased simulations for the calculation of fast-timescale order parameters. We also performed 28 independent unbiased MD simulations, each $50 \mathrm{~ns}$ long, at 304K and with the same computational setup as the restrained simulations, but without any restraints. As starting points, we selected seven different structures from each of the four replicas obtained in the CS-NOE-4 ensemble (Table S1). In particular, the seven structures were selected from the SA cycles after convergence (i.e. at SA cycles $65,75,85,95,100,110,125$ ). We calculated fast timescale order parameters, which correspond to those measured by NMR relaxation measurements, from these 28 unbiased simulations using a previously described approach (Maragakis et al., 2008). In particular, we calculated bond-vector autocorrelation functions (independently from each simulation) including both internal motions and overall tumbling of NCBD. The resulting correlation functions were then averaged over the 28 simulations and subsequently fitted globally to a Lipari-Szabo model (Lipari \& Szabo, 1982) to yield relaxation order parameters. To calculate order parameters that report on the long-timescale motions we first aligned the full ensemble and then calculated order parameters as ensemble averages (Maragakis et al., 2008).

Analyses of convergence and cross validation. We used two different methods to examine the convergence of our simulations. First, we used the ENCORE ensemble comparison method (Lindorff-Larsen \& Ferkinghoff-Borg, 2009; Tiberti et al., 2015) to quantify the overlap between the structural ensembles. The latter is based on clustering the structures using affinity propagation (setting the "preference value" in the clustering to 12) and subsequent comparison of the ensembles by calculating the Jensen-Shannon (JS) divergence between pairs of ensembles by comparing how they populate the different clusters. For additional details, please confer to original descriptions of the method (Lindorff-Larsen \& Ferkinghoff-Borg, 2009; Tiberti et al., 2015). As an alternative method, we calculated the Root Mean Square Inner Product (RMSIP) over the first 10 eigenvectors obtained from a principal component analysis of the covariance matrix of atomic $\left(\mathrm{C}_{\alpha}\right.$-atoms $)$ fluctuations (Amadei, Linssen \& Berendsen, 1993). 
236

237

238

239

240

241

242

243

244

245

246

247

248

249

250

251

252

253

254

255

256

257

258

259

260

261

262

263

264

265

To cross-validate our ensembles we calculated the chemical shifts of side chain methyl hydrogen and carbon atoms using CH3Shift (Sahakyan et al., 2011) (both ${ }^{1} \mathrm{H}$ and ${ }^{13} \mathrm{C}$ shifts) and PPM (Li \& Brüschweiler, 2012) (only ${ }^{1} \mathrm{H}$ shifts) and compared to the previously determined experimental side chain chemical shifts. In particular, we compared the calculated side chain chemical shifts with the experimental values (deposited in BMRB entry 16363) using a reduced $\chi^{2}$ metric. In this metric, the square deviation between the calculated and experimental values were normalized by the variance of the chemical shift predictor (for each type of chemical shift) and the total number of chemical shifts, so that low numbers indicate good agreement between experimental and calculated chemical shifts.

\section{RESULTS}

Convergence of the simulations. Before assessing the accuracy of the different structural ensembles that we generated, we first ensured that the simulated annealing protocol allowed us to obtain converged ensembles that represent the dynamical properties encoded in the experimental restraints and the molecular force field. To quantify convergence of the ensembles, we calculated two different measures of the overlap between the subspaces sampled by different simulations.

First, we used a previously described approach (Lindorff-Larsen \& Ferkinghoff-Borg, 2009; Tiberti et al., 2015), which is based on a quantification of the extent to which the different ensembles mix during conformational clustering, to calculate the Jensen-Shannon (JS) divergence between the ensembles (Figure 2). A JS divergence of zero is evidence of identical ensembles, and it has previously been observed that a JS divergence in the range of 0.1-0.3 represents similar ensembles (Lindorff-Larsen \& Ferkinghoff-Borg, 2009; Tiberti et al., 2015). We expect that in a converged replica-averaged simulation that the different replicas should populate equally the different structural basins. With this in mind, we calculated the JS divergence between two replicas in a simulation restrained by NOEs and chemical shifts (Figure 2, black line). We find that after approximately $\sim 30$ cycles of simulated annealing the two replicas have covered approximately the same conformational space with the JS divergence stabilizing around 0.2-0.3 with the fluctuations in the JS-divergence representing the stochastic nature of the simulations. Thus, we decided to discard the first 45 simulated annealing cycles from all the simulations. As an alternative measure of ensemble similarity we also calculated the Root Mean Square Inner Product (Hess, 2002) 
266 (RMSIP) with very similar results. In particular, the similarity of the two replicas converge to an

267 RMSIP value greater than 0.83 (here RMSIP=1 is expected for fully overlapping ensembles).

268 As a second, perhaps even more stringent, test of convergence we also examined whether two 269 simulations with the same number of replicas and experimental restraints, but initiated from 270 substantially different starting structures, converge to similar ensembles. Indeed, we find that 271 simulations initiated from two distinct structures of NCBD (Table S1) converge to similar 272 ensembles when the first 45 cycles are discarded as initial equilibration (Figure 2, grey line). Thus, 273 based on these two tests we concluded that our sampling protocol allows us to obtain structural 274 ensembles that represent the force field and restraints employed.

275 Assessment of the accuracy of the NCBD ensembles. Once we had assessed the convergence 276 of the simulations, we analysed the different ensembles to evaluate their accuracy. To do so, we 277 back-calculated experimental parameters that were not used as restraints and compared them with 278 the experimental values. As our different simulations employed different sets of experimental 279 restraints, not all experimental data can be employed for validation purposes. For example, while 280 the NOEs can be used to evaluate the quality of an ensemble obtained using CS-restraints, they 281 can obviously not be used to validate an ensemble that was generated using those NOEs as 282 restraints.

283 We first examined whether the CS or NOE restraints alone are sufficient to increase the accuracy 284 in the description of the conformational ensemble of NCBD. We thus compared unbiased 285 simulations with simulations biased by either CS or NOEs by cross-validation with the measured 286 NOEs and CS, respectively.

287 We back-calculated NOEs from the inter-proton distances and observed substantial violations 288 (some greater than $2 \AA$ ) in both unbiased and CS ensembles (Figure S1) independently of the 289 number of replicas used for the averaging. To determine the origin of these discrepancies we 290 calculated intramolecular contacts between side chains, and observed an overall decrease in these 291 (from 27 in the previously-determined NMR ensemble, to 14 and 17 in unbiased and CS292 restrained, respectively). More specifically we found a loss of inter-helical contacts between 293 helices $\alpha 1$ and $\alpha 2$ in the simulations, in agreement with our finding of several long-range NOEs 294 that are violated in these ensembles. 
295

296

297

298

299

300

301

302

303

304

305

306

307

308

309

310

311

312

313

314

315

316

317

318

319

320

321

322

323

324

325

These results demonstrate that the CS-restraints and MD force field, as implemented here, are not sufficient to provide a fully accurate description of the conformational ensemble of NCBD. Similarly, we found that back-calculation of backbone chemical shifts from the unbiased simulation and, to a lesser extent a NOE-restrained ensemble, resulted in deviations from experiments. We therefore decided to determine conformational ensembles that combine the information of the NOEs, chemical shifts and force field in replica-averaged simulations (CSNOE) aiming to provide a more accurate structural ensemble of NCBD than possible via the application of just one of the two classes of restraints. We also assessed the influence of the choice of force field since we expected that a more accurate ensemble could be obtained with the relatively limited amounts of experimental data when using a more accurate force field. Thus, we compared simulations using either the CHARMM22 force field (CS-NOE-4-C22 simulation), or a more recent and accurate force field variant, CHARMM22* (CS-NOE simulations).

As both the NOEs and backbone chemical shifts were used as restraints they cannot be used for validation of these ensembles. Instead, we turned to side-chain methyl chemical shifts for a comparison and validation of the different ensembles. Methyl-containing residues, for which the chemical shifts are available, cover the entire protein structure and are thus excellent probes of both local structure $\left({ }^{13} \mathrm{C}\right.$ methyl chemical shifts, which are mostly dependent on the rotameric state) and long-range contacts $\left({ }^{1} \mathrm{H}\right.$ methyl chemical shifts). The methyl chemical shifts were predicted by CH3Shift (Sahakyan et al., 2011) and the resulting values compared to experiments, separating the contributions from ${ }^{13} \mathrm{C}$ and ${ }^{1} \mathrm{H}$. We then calculated $\chi^{2}$ red thus taking into account the inherent uncertainty of the chemical shift predictions (Sahakyan et al., 2011).

As also indicated by the calculation of NOEs and backbone chemical shifts, we find that the side chain chemical shifts predicted from the unbiased simulation (green line in Figure 3) deviates substantially from experiments. The introduction of backbone chemical shift restraints (CS ensembles, orange line in Figure 3) provides a better structural ensemble than the force field alone, especially for ${ }^{13} \mathrm{C}$ methyl chemical shifts and when averaged over 2 or 4 replicas. We also calculated the chemical shifts from NOE-derived ensembles, obtained with or without replicaaveraging. Surprisingly, we find that the ensembles obtained using NOEs as replica-averaged restraints (NOE, magenta line in Figure 3) perform slightly worse than the CS ensemble. Thus, when evaluated in this way, ensembles derived by MD refinement using either backbone chemical shifts or NOEs do not increase accuracy compared to the ensemble deposited in the PDB. 
326 By combining the NOEs, chemical shifts and the CHARMM22* force field we were, however, 327 able to obtain even more accurate ensembles, in particular when averaging over four replicas, as 328 assessed by the ability to predict side chain ${ }^{13} \mathrm{C}$ and ${ }^{1} \mathrm{H}$ methyl chemical shifts (Figure 3). 329 Interestingly we find that not only the experimental data but also the CHARMM22* force field 330 contributes to the improved agreement with the experimental data. Indeed, when we employ both 331 chemical shift and NOE-based restraints in simulations averaged over 4 replicas, but replacing the 332 CHARMM22* force field by an earlier, less accurate variant of the same force field 333 (CHARMM22; CS-NOE-4-C22) (Lindorff-Larsen et al., 2012a) we find that the accuracy 334 decreases dramatically. Calculations of ${ }^{1} \mathrm{H}$ methyl chemical shifts using PPM (Li \& Brüschweiler, 335 2012) instead of $\mathrm{CH} 3$ Shift demonstrate that the conclusions are robust to the method for calculating 336 the chemical shifts (Figure S2). Similarly, calculations of the chemical shifts using the ensemble 337 generated from the alternative starting structure (CS-NOE-2-1ZOQ) resulted in essentially the 338 same agreement with the experimental data as when simulations were initiated from the $2 \mathrm{KKJ}$ 339 structure (Figure 3), confirming the conclusions from the convergence analysis described above 340 (Figure 2). The CS-NOE-4 ensemble, which we found to provide the most accurate representation 341 of the free state of NCBD in solution, is shown in Figure 4. It is a relatively broad ensemble of 342 conformations, where the three helical regions are maintained overall, but differ in the lengths and 343 relative positions of the three $\alpha$-helices.

344 Small Angle X-ray scattering (SAXS) measurements have been carried out for NCBD in solution 345 (Kjaergaard, Teilum \& Poulsen, 2010) and previously been compared to simulation-derived ensembles of NCBD (Knott \& Best, 2012; Naganathan \& Orozco, 2013). We thus calculated the radius of gyration $\left(R_{g}\right)$ using CRYSOL (Svergun, Barberato \& Koch, 1995) for the various ensembles. In all cases we find that the average $R_{g}$ values are in the range of $13.7 \AA-14.9 \AA$. These values are comparable to that obtained previously from simulations (13.7 $\AA$ ) (Knott \& Best, 2012) but lower than the values estimated from a Guinier analysis of the experimental data $(\sim 16.5 \AA)$ or an ensemble-optimization method (18.8 A) (Kjaergaard, Teilum \& Poulsen, 2010). We note,

352 however, that the experimental values also include contributions from a $\sim 8 \%$ population of unfolded protein that is not captured by our simulations. Although a detailed understanding is

354 lacking for the role of solvation on the SAXS properties of partially disordered proteins we,

355 however, expect that the discrepancy between experiment and simulation should be ascribed to 356 remaining force field deficiencies. Indeed, overly large compaction of proteins is a common 
357 problem of most atomistic force fields (Piana, Klepeis \& Shaw, 2014) though recent work suggests 358 that, at least for fully disordered proteins, that modified protein-water interactions can improve accuracy (Nerenberg et al., 2012; Best, Zheng \& Mittal, 2014; Henriques, Cragnell \& Skepö, 2015;

360 Mercadante et al., 2015; Piana et al., 2015). We also note that while the force field used here 361 (CHARMM22*) in certain cases has been shown to produce too compact structures, (Piana et al., 362 2015) in other cases it appears to perform quite well (Rauscher et al., 2015). We expect that 363 resolving these issues will require both further force field developments (Best, 2017) as well as improved methods for comparing experiments and SAXS experiments (Hub, 2018).

A unified view of NCBD dynamics. While the broad peaks and sparse NOEs are suggestive of a rather dynamic protein, previous NMR relaxation measurements of side chain dynamics found relatively high order parameters $\left(S_{\text {relaxation }}\right)$ comparable to values found in well-ordered proteins (Kjaergaard, Poulsen \& Teilum, 2012). To shed light on this apparent discrepancy and to assess whether our relatively broad structural ensemble is compatible with mobility on different timescales, we calculated $S^{2}$ values representing different timescales.

371 To mimic the dynamics probed in relaxation experiments we selected 28 structures from each of the 4 replicas of the CS-NOE-4 ensemble sampled at seven different SA steps. Starting from each of these conformations we performed $50 \mathrm{~ns}$ of unbiased MD simulation (in total $1.4 \mu \mathrm{s}$, Figure

374 S3), and from each simulation we calculated the autocorrelation functions of the N-H bond vectors (without removing the overall rotational motion of the protein). These correlation functions were subsequently averaged and fitted to the Lipari-Szabo model to estimate the $S_{\text {relaxation }}^{2}$ values, which report on the nanosecond dynamics of the protein (Figure 5, black line). The results show a relatively rigid ensemble on the ns timescale attested by high order parameters throughout most of the polypeptide backbone.

To quantify the backbone dynamics on the longer timescales that may influence both the NOE and chemical shifts (but which the relaxation measurements would not be sensitive to) we defined and calculated " $S^{2}$ chemical shift"-values from the structural variability in the ensemble after aligning the structures. These $S^{2}$ values include contributions also from any millisecond-timescale motions that might be present in the ground state of NCBD. As internal and overall motions cannot be decoupled, the results of such calculations will depend on how the ensemble is aligned. In our calculations we chose THESEUS (Theobald \& Steindel, 2012) as the least biased method to align the structures (Figure 4). These order parameter calculations reveal a broader distribution of 
388 conformations with additional, longer-timescale dynamics evident both in loop regions and the C-

389 terminal region, even though relatively high $S^{2}$ values are found in the regions of secondary

390 structures (Figure 5, grey line).

391 A similar analysis of side chain motions suggests even greater differences in motions present on 392 relaxation and chemical shift timescales. In particular, we find that, for methyl-bearing side chains,

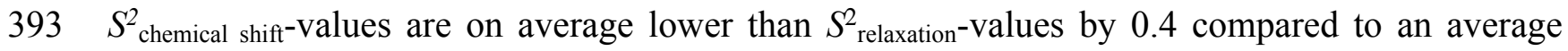
394 difference of 0.2 for the backbone amides. Finally, we note that although both calculated $\mathrm{S}^{2}$ chemical

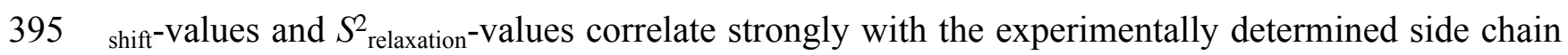

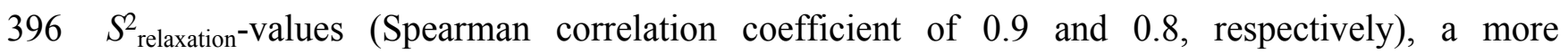
397 quantitative analysis is hampered by several issues including (i) the presence of a small population 398 of unfolded protein in the experiments, (ii) the difficulty in appropriate model selection of the 399 calculated correlation functions, (iii) the well-known observation of too-fast rotational motions of 400 proteins in the TIP3P model that we used and (iv) uncertainties in the parameterization of the 401 rotational motions in the experimental analyses. We note, however, the potential complications 402 that arise from the fact that the $S^{2}$ chemical shift-values were obtained from simulations with an

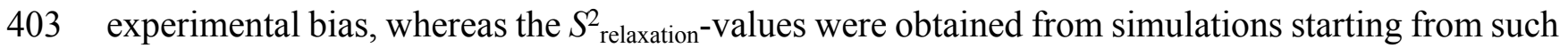
404 a biased ensemble, but performed with the standard CHARMM22* force field.

405

\section{DISCUSSION}

407 We find that after approximately $~ 30$ cycles of simulated annealing that two "identical" replicas 408 have covered approximately the same conformational as judged by the JS divergence between 409 them. Similarly, we find that simulations initiated from two distinct structures of NCBD converge 410 to similar ensembles when the first 45 cycles are discarded. Thus, based on these two tests we 411 concluded that our sampling protocol allows us to obtain structural ensembles that represent the 412 force field and restraints employed.

413 Once we had assessed the convergence of the simulations, we analysed the different ensembles 414 to evaluate their accuracy. As our different simulations employed different sets of experimental 415 restraints, not all experimental data can be employed for validation purposes.

416 Our results revealed that the CS-restraints and MD force field, as implemented here, are not 417 alone enough to describe accurately the conformational ensemble of NCBD. We therefore 
418 determined conformational ensembles that combine the information of the NOEs, chemical shifts 419 and force field, and validated them using side-chain methyl chemical shifts. The results show that 420 by combining the NOEs, chemical shifts and the CHARMM22* force field we are able to obtain 421 even more accurate ensembles (compared to using these data individually), in particular when 422 averaging over four replicas. Thus, we find that the CS-NOE-4 ensemble provides the most 423 accurate representation of the free state of NCBD in solution among the different ensembles we 424 have studied. We, however, find that this ensemble is slightly more compact than expected from 425 experiment, and suggest that a more careful analysis of the SAXS data and a force field that gives 426 a better balance between compact and expanded structures are necessary to solve these issues.

427 Our results also shed new light on the amount and time-scales of the dynamics in NCBD. In 428 particular, our calculations of order parameters demonstrate that NCBD may be described as a 429 semi-rigid protein on fast-timescales, but with additional dynamics in the backbone and-in 430 particular - side chains on timescales longer than the rotational correlation time of the protein, as 431 also previously suggested (Kjaergaard, Poulsen \& Teilum, 2012).

432

\section{CONCLUSIONS}

434 We have presented an application of the dynamic-ensemble refinement method to study the 435 native state dynamics of NCBD. In the original implementation of DER we combined NMR relaxation order parameters with NOEs in MD simulations (Lindorff-Larsen et al., 2005). This approach was here extended to the combination of chemical shifts and NOEs to make it more generally applicable. In particular, our results show that it is possible to combine NOEs, backbone chemical shifts and an accurate MD force field into replica-averaged restrained simulations, and that all three components add substantially to the accuracy of the resulting NCBD ensemble.

441 NMR structures are typically obtained by combining distance information from NOE measurements with in vacuo simulations, in certain cases with subsequent refinement by short, MD simulations in explicit solvent. Further, the inherent ensemble averaging of the experimental data is typically not exploited explicitly. In this way, standard NMR structures can provide highly accurate models of the "average structure" of a protein, but only little information about the conformational heterogeneity around this average. 
447

448

449

450

451

452

453

454

455

456

457

458

459

460

461

462

463

464

465

466

467

468

469

470

471

472

473

474

475

476

Replica-averaged MD simulations make it possible to obtain structural ensembles that match the experimental data according to the principle of maximum entropy (Pitera \& Chodera, 2012; Roux \& Weare, 2013; Cavalli, Camilloni \& Vendruscolo, 2013; Boomsma, Ferkinghoff-Borg \& Lindorff-Larsen, 2014; White \& Voth, 2014; Olsson et al., 2014). In such calculations prior information, here in the form of a molecular mechanics force field, is biased in a minimal fashion to agree with the experimental data. Thus, to obtain an accurate ensemble, such simulations require an accurate force field, an efficient sampling approach as well as sufficient experimental information. Our results show that, at least in the case of the small, but relatively mobile protein NCBD, it is possible to perform such simulations when NOEs are supplemented by the information available in the backbone chemical shifts and a well-parameterized molecular force field. The application of the experimentally-derived structural restraints helps overcome at least some of the deficiencies in force field accuracy and also improves sampling of the relevant regions of conformational space. While we find that four replicas are optimal for the system and data studied here, we expect that this value might vary between systems and hence recommend evaluating it e.g. via comparing to independently measured data such as the side chain shifts analysed here.

Our approach also allowed us to probe the structural heterogeneity arising from both short- and long-timescale dynamics by the calculation of order parameters. In the case of NCBD we found that this protein can be described as a relatively rigid protein domain on a fast timescale, as attested by the high relaxation order parameters that, nevertheless, displays additional motions in both the backbone and side chains on longer timescales. This situation is reminiscent of the molten globule state of apomyoglobin, that also displays restricted motions on the nanosecond timescale but with greater motions on a slower timescale (Eliezer et al., 2000; Meinhold \& Wright, 2011). The current study also provides the groundwork for further studies on NCBDs intricate conformational dynamics, and the relationship to ligand binding (Dogan et al., 2012; Zijlstra et al., 2017). Given the importance of understanding and quantifying protein dynamics, in particular on long timescales, we expect that our approach, which uses only commonly available data, and possible combined with novel algorithms for enhancing sampling (Bonomi et al., 2016; Bonomi, Camilloni \& Vendruscolo, 2016), will have a wide range of applications.

\section{7 \\ Acknowledgements}


478 We would like to thank Magnus Kjaergaard, Wouter Boomsma, Matteo Tiberti and Peter Wright 479 for fruitful discussion and comments.

\section{Supporting Information}

481 Supporting figures with the data on back-calculation of NOEs in MD-unbiased and MD-CS 482 ensembles are reported in Figure S1. The deviation between experimental and calculated side483 chain ${ }^{1} \mathrm{H}$ chemical shifts calculated with PPM software are shown in Figure S2. The main-chain 484 RMSD profiles of the 28 unbiased constant temperature MD simulations of NCBD are reported in 485 Figure S3. The online raw data contains the experimental data used in our simulations as well as 486 scripts to perform the simulations using Gromacs and PLUMED. 
488

489

490

491

492

493

494

495

496

497

498

499

500

501

502

503

504

505

506

507

508

509

510

511

512

513

514

515

\section{REFERENCES}

Amadei A., Linssen AB., Berendsen HJ. 1993. Essential dynamics of proteins. Proteins 17:41225. DOI: $10.1002 /$ prot.340170408.

Ángyán AF., Gáspári Z. 2013. Ensemble-based interpretations of NMR structural data to describe protein internal dynamics. Molecules 18:10548-10567. DOI: 10.3390/molecules 180910548.

Best RB. 2017. Computational and theoretical advances in studies of intrinsically disordered proteins. Current Opinion in Structural Biology 42:147-154. DOI: 10.1016/j.sbi.2017.01.006.

Best RB., Zheng W., Mittal J. 2014. Balanced Protein - Water Interactions Improve Properties of Disordered Proteins and Non-Specific Protein Association. Journal of Chemical Theory and Computation 10:5113-5124.

Bonomi M., Branduardi D., Bussi G., Camilloni C., Provasi D., Raiteri P., Donadio D., Marinelli F., Pietrucci F., Broglia RA., Parrinello M. 2009. PLUMED: A portable plugin for freeenergy calculations with molecular dynamics. Computer Physics Communications 180:19611972. DOI: 10.1016/j.cpc.2009.05.011.

Bonomi M., Camilloni C., Cavalli A., Vendruscolo M. 2016. Metainference: A Bayesian inference method for heterogeneous systems. Science Advances 2:e1501177-e1501177. DOI: 10.1126/sciadv. 1501177 .

Bonomi M., Camilloni C., Vendruscolo M. 2016. Metadynamic metainference: Enhanced sampling of the metainference ensemble using metadynamics. Scientific Reports 6:31232. DOI: $10.1038 /$ srep31232.

Bonomi M., Heller GT., Camilloni C., Vendruscolo M. 2017. Principles of protein structural ensemble determination. Current Opinion in Structural Biology 42:106-116. DOI: 10.1016/J.SBI.2016.12.004.

Boomsma W., Ferkinghoff-Borg J., Lindorff-Larsen K. 2014. Combining experiments and simulations using the maximum entropy principle. PLoS computational biology 10:e1003406. DOI: 10.1371/journal.pcbi.1003406.

Boomsma W., Tian P., Frellsen J., Ferkinghoff-Borg J., Hamelryck T., Lindorff-Larsen K., 
516

517

518

519

520

521

522

523

524

525

526

527

528

529

530

531

532

533

534

535

536

537

538

539

540

541

542

543

544

Vendruscolo M. 2014. Equilibrium simulations of proteins using molecular fragment replacement and NMR chemical shifts. Proceedings of the National Academy of Sciences of the United States of America 111:13852-7. DOI: 10.1073/pnas.1404948111.

Bottaro S., Bussi G., Kennedy SD., Turner DH., Lindorff-Larsen K. 2018. Conformational ensembles of RNA oligonucleotides from integrating NMR and molecular simulations. Science Advances 4:eaar8521 DOI: 10.1126/sciadv.aar8521

Bussi G., Donadio D., Parrinello M. 2007. Canonical sampling through velocity rescaling. The Journal of chemical physics 126:14101. DOI: 10.1063/1.2408420.

Camilloni C., Cavalli A., Vendruscolo M. 2013a. Assessment of the Use of NMR Chemical Shifts as Replica-Averaged Structural Restraints in Molecular Dynamics Simulations to Characterise the Dynamics of Proteins. The journal of physical chemistry. B. DOI: $10.1021 /$ jp3106666.

Camilloni C., Cavalli A., Vendruscolo M. 2013b. Replica-Averaged Metadynamics. Journal of Chemical Theory and Computation 9:5610-5617. DOI: 10.1021/ct4006272.

Camilloni C., Robustelli P., De Simone A., Cavalli A., Vendruscolo M. 2012. Characterisation of the conformational equilibrium between the two major substates of RNase A using NMR chemical shifts. Journal of the American Chemical Society 134:3968-3971.

Camilloni C., Vendruscolo M. 2014. Statistical mechanics of the denatured state of a protein using replica-averaged metadynamics. Journal of the American Chemical Society 136:8982-91. DOI: $10.1021 /$ ja5027584.

Cavalli A., Camilloni C., Vendruscolo M. 2013. Molecular dynamics simulations with replicaaveraged structural restraints generate structural ensembles according to the maximum entropy principle. The Journal of chemical physics 138:94112. DOI: 10.1063/1.4793625.

Cavalli A., Salvatella X., Dobson CM., Vendruscolo M. 2007. Protein structure determination from NMR chemical shifts. Proceedings of the National Academy of Sciences of the United States of America 104:9615-20. DOI: 10.1073/pnas.0610313104.

Demarest SJ., Deechongkit S., Dyson HJ., Evans RM., Wright PE. 2004. Packing, specificity, and mutability at the binding interface between the p160 coactivator and CREB-binding protein. Protein science 13:203-10. DOI: 10.1110/ps.03366504. 
545 Demarest SJ., Martinez-Yamout M., Chung J., Chen H., Xu W., Dyson HJ., Evans RM., Wright 546 PE. 2002. Mutual synergistic folding in recruitment of CBP/p300 by p160 nuclear receptor coactivators. Nature 415:549-53. DOI: 10.1038/415549a.

Dogan J., Schmidt T., Mu X., Engström Å., Jemth P. 2012. Fast association and slow transitions in the interaction between two intrinsically disordered protein domains. The Journal of biological chemistry 287:34316-24. DOI: 10.1074/jbc.M112.399436.

Dror RO., Dirks RM., Grossman JP., Xu H., Shaw DE. 2012. Biomolecular simulation: a 552 computational microscope for molecular biology. Annual review of biophysics 41:429-52. DOI: 10.1146/annurev-biophys-042910-155245.

Ebert M-O., Bae S-H., Dyson HJ., Wright PE. 2008. NMR relaxation study of the complex formed between CBP and the activation domain of the nuclear hormone receptor coactivator ACTR. Biochemistry 47:1299-308. DOI: 10.1021/bi701767j.

Eliezer D., Chung J., Dyson HJ., Wright PE. 2000. Native and Non-native Secondary Structure and Dynamics in the pH 4 Intermediate of Apomyoglobin. Biochemistry 39:2894-2901. DOI: 10.1021/BI992545F.

Essmann U., Perera L., Berkowitz ML., Darden T., Lee H., Pedersen LG. 1995. A smooth particle 561 mesh Ewald method. The Journal of Chemical Physics 103:8577. DOI: 10.1063/1.470117.

562 563

Esteban-Martín S., Bryn Fenwick R., Salvatella X. 2012. Synergistic use of NMR and MD simulations to study the structural heterogeneity of proteins. Wiley Interdisciplinary Reviews: Computational Molecular Science 2:466-478. DOI: 10.1002/wcms.1093.

Fenwick RB., Esteban-Martín S., Richter B., Lee D., Walter KFA., Milovanovic D., Becker S., Lakomek NA., Griesinger C., Salvatella X. 2011. Weak Long-Range Correlated Motions in a Surface Patch of Ubiquitin Involved in Molecular Recognition. Journal of the American Chemical Society 133:10336-10339. DOI: 10.1021/ja200461n.

Fiser A., Šali A. 2003. MODELLER: Generation and Refinement of Homology-Based Protein Structure Models. Methods in Enzymology 374:461-491. DOI: 10.1016/S00766879(03)74020-8.

Han B., Liu Y., Ginzinger SW., Wishart DS. 2011. SHIFTX2: significantly improved protein chemical shift prediction. Journal of biomolecular NMR 50:43-57. DOI: 10.1007/s10858- 
011-9478-4.

575 Henriques J., Cragnell C., Skepö M. 2015. Molecular dynamics simulations of intrinsically 576 disordered proteins: force field evaluation and comparison with experiment. Journal of Chemical Theory and Computation 11:3420-3431. DOI: 10.1021/ct501178z.

Hess B. 2002. Convergence of sampling in protein simulations. Physical review. E, Statistical, nonlinear, and soft matter physics 65:31910.

Hess B., Bekker H., Berendsen H., Fraaije J. 1993. LINCS: A linear constraint solver for molecular simulations. Journal of Computational Chemistry 12:1463-1472. DOI: 10.1002/(SICI)1096987X(199709)18:12\&lt;1463::AID-JCC4\&gt;3.0.CO;2-H.

Hub JS. 2018. Interpreting solution X-ray scattering data using molecular simulations. Current

585 Opinion in Structural Biology 49:18-26. DOI: 10.1016/J.SBI.2017.11.002.

Hummer G., Köfinger J. 2015. Bayesian ensemble refinement by replica simulations and reweighting. The Journal of chemical physics 143:243150. DOI: 10.1063/1.4937786.

Islam SM., Stein RA., McHaourab HS., Roux B. 2013. Structural refinement from restrainedensemble simulations based on EPR/DEER data: application to T4 lysozyme. The journal of physical chemistry. B 117:4740-54. DOI: 10.1021/jp311723a.

Jorgensen WL., Chandrasekhar J., Madura JD., Impey RW., Klein ML. 1983. Comparison of simple potential functions for simulating liquid water. The Journal of Chemical Physics 79:926. DOI: 10.1063/1.445869.

Kannan A., Camilloni C., Sahakyan AB., Cavalli A., Vendruscolo M. 2014. A conformational ensemble derived using NMR methyl chemical shifts reveals a mechanical clamping transition that gates the binding of the HU protein to DNA. Journal of the American Chemical Society 136:2204-7. DOI: 10.1021/ja4105396.

Kjaergaard M., Andersen L., Nielsen LD., Teilum K. 2013. A Folded Excited State of LigandFree Nuclear Coactivator Binding Domain (NCBD) Underlies Plasticity in Ligand Recognition. Biochemistry:130201143825004. DOI: 10.1021/bi4001062.

Kjaergaard M., Poulsen FM., Teilum K. 2012. Is a malleable protein necessarily highly dynamic? The hydrophobic core of the nuclear coactivator binding domain is well ordered. Biophysical 

journal 102:1627-35. DOI: 10.1016/j.bpj.2012.02.014.

603 Kjaergaard M., Teilum K., Poulsen FM. 2010. Conformational selection in the molten globule 604 state of the nuclear coactivator binding domain of CBP. Proceedings of the National Academy 605 of Sciences of the United States of America 107:12535-40. DOI: 10.1073/pnas.1001693107.

606 Knott M., Best RB. 2012. A preformed binding interface in the unbound ensemble of an 607 intrinsically disordered protein: evidence from molecular simulations. PLoS computational 608 biology 8:e1002605. DOI: 10.1371/journal.pcbi.1002605.

Kohlhoff KJ., Robustelli P., Cavalli A., Salvatella X., Vendruscolo M. 2009. Fast and accurate predictions of protein NMR chemical shifts from interatomic distances. Journal of the American Chemical Society 131:13894-5. DOI: 10.1021/ja903772t.

Krieger JM., Fusco G., Lewitzky M., Simister PC., Marchant J., Camilloni C., Feller SM., De Simone A. 2014. Conformational recognition of an intrinsically disordered protein. Biophysical journal 106:1771-9. DOI: 10.1016/j.bpj.2014.03.004.

Kukic P., Camilloni C., Cavalli A., Vendruscolo M. 2014. Determination of the Individual Roles of the Linker Residues in the Interdomain Motions of Calmodulin Using NMR Chemical Shifts. Journal of molecular biology 426:1826-1838. DOI: 10.1016/j.jmb.2014.02.002.

Lange OF., Lakomek N-A., Farès C., Schröder GF., Walter KFA., Becker S., Meiler J., Grubmüller H., Griesinger C., de Groot BL. 2008. Recognition dynamics up to microseconds revealed from an RDC-derived ubiquitin ensemble in solution. Science (New York, N.Y.) 320:1471-5. DOI: 10.1126/science.1157092.

Lee CW., Martinez-Yamout MA., Dyson HJ., Wright PE. 2010. Structure of the p53 transactivation domain in complex with the nuclear receptor coactivator binding domain of CREB binding protein. Biochemistry 49:9964-71. DOI: 10.1021/bi1012996.

Lehtivarjo J., Tuppurainen K., Hassinen T., Laatikainen R., Peräkylä M. 2012. Combining NMR ensembles and molecular dynamics simulations provides more realistic models of protein structures in solution and leads to better chemical shift prediction. Journal of biomolecular NMR 52:257-67. DOI: 10.1007/s10858-012-9609-6.

Li D-W., Brüschweiler R. 2012. PPM: a side-chain and backbone chemical shift predictor for the assessment of protein conformational ensembles. Journal of biomolecular NMR 54:257-65. 
632 Lindorff-Larsen K., Best RB., Depristo MA., Dobson CM., Vendruscolo M. 2005. Simultaneous 633 determination of protein structure and dynamics. Nature 433:128-32. DOI: $634 \quad 10.1038 /$ nature03199.

635 Lindorff-Larsen K., Ferkinghoff-Borg J. 2009. Similarity measures for protein ensembles. PloS 636 one 4:e4203. DOI: 10.1371/journal.pone.0004203.

637 Lindorff-Larsen K., Maragakis P., Piana S., Eastwood MP., Dror RO., Shaw DE. $2012 \mathrm{a}$. 638 Systematic validation of protein force fields against experimental data. PloS one 7:e32131. 639 DOI: 10.1371/journal.pone.0032131.

640 Lindorff-Larsen K., Trbovic N., Maragakis P., Piana S., Shaw DE. 2012b. Structure and dynamics 641 of an unfolded protein examined by molecular dynamics simulation. Journal of the American 643 Chemical Society 134:3787-91. DOI: 10.1021/ja209931w.

Lipari G., Szabo A. 1982. Model-free approach to the interpretation of nuclear magnetic resonance 644 relaxation in macromolecules. 1. Theory and range of validity. Journal of the American Chemical Society 104:4546-4559. DOI: 10.1021/ja00381a009.

Löhr T., Jussupow A., Camilloni C. 2017. Metadynamic metainference: Convergence towards 647 force field independent structural ensembles of a disordered peptide. The Journal of Chemical Physics 146:165102. DOI: 10.1063/1.4981211.

MacCallum JL., Perez A., Dill KA. 2015. Determining protein structures by combining 650 semireliable data with atomistic physical models by Bayesian inference. Proceedings of the National Academy of Sciences of the United States of America 112:6985-90. DOI: 10.1073/pnas. 1506788112 .

MacKerell, AD., Bashford D., Dunbrack, RL., Evanseck JD., Field MJ., Fischer S., Gao J., Guo H., Ha S., Joseph-McCarthy D., Kuchnir L., Kuczera K., Lau FTK., Mattos C., Michnick S., Ngo T., Nguyen DT., Prodhom B., Reiher WE., Roux B., Schlenkrich M., Smith JC., Stote R., Straub J., Watanabe M., Wiórkiewicz-Kuczera J., Yin D., Karplus M. 1998. All-Atom of Physical Chemistry B 102:3586-3616. DOI: 10.1021/jp973084f.

Maragakis P., Lindorff-Larsen K., Eastwood MP., Dror RO., Klepeis JL., Arkin IT., Jensen MØ., 
660

661

662

663

664

665

666

667

668

669

670

671

672

673

674

675

676

677

678

679

680

681

682

683

684

685

686

687

688

Xu H., Trbovic N., Friesner RA., Palmer AG., Shaw DE. 2008. Microsecond Molecular Dynamics Simulation Shows Effect of Slow Loop Dynamics on Backbone Amide Order Parameters of Proteins. The Journal of Physical Chemistry B 112:6155-6158. DOI: 10.1021/jp077018h.

Meinhold DW., Wright PE. 2011. Measurement of protein unfolding/refolding kinetics and structural characterization of hidden intermediates by NMR relaxation dispersion. Proceedings of the National Academy of Sciences of the United States of America 108:907883. DOI: 10.1073/pnas.1105682108.

Mercadante D., Milles S., Fuertes G., Svergun DI., Lemke E a., Gräter F. 2015. Kirkwood-Buff approach rescues over-collapse of a disordered protein in canonical protein force fields. The Journal of Physical Chemistry B:150601163019003. DOI: 10.1021/acs.jpcb.5b03440.

Mobley DL. 2012. Let's get honest about sampling. Journal of computer-aided molecular design 26:93-5. DOI: 10.1007/s10822-011-9497-y.

Naganathan AN., Orozco M. 2013. The conformational landscape of an intrinsically disordered DNA-binding domain of a transcription regulator. The journal of physical chemistry. B 117:13842-50. DOI: 10.1021/jp408350v.

Nerenberg PS., Jo B., So C., Tripathy A., Head-Gordon T. 2012. Optimizing solute-water van der Waals interactions to reproduce solvation free energies. The journal of physical chemistry. $B$ 116:4524-34. DOI: 10.1021/jp2118373.

Olsson S., Vögeli BR., Cavalli A., Boomsma W., Ferkinghoff-Borg J., Lindorff-Larsen K., Hamelryck T. 2014. Probabilistic Determination of Native State Ensembles of Proteins. Journal of Chemical Theory and Computation 10:3484-3491. DOI: 10.1021/ct5001236.

Papaleo E., Sutto L., Gervasio FL., Lindorff-Larsen K. 2014. Conformational Changes and Free Energies in a Proline Isomerase. Journal of Chemical Theory and Computation 10:41694174. DOI: $10.1021 / \mathrm{ct} 500536$ r.

Perilla JR., Goh BC., Cassidy CK., Liu B., Bernardi RC., Rudack T., Yu H., Wu Z., Schulten K. 2015. Molecular dynamics simulations of large macromolecular complexes. Current Opinion in Structural Biology 31:64-74. DOI: 10.1016/j.sbi.2015.03.007.

Piana S., Donchev AG., Robustelli P., Shaw DE. 2015. Water Dispersion Interactions Strongly 
689

690

691

692

693

694

695

696

697

698

699

700

701

702

703

704

705

706

707

708

709

710

711

712

713

714

715

716

717

Influence Simulated Structural Properties of Disordered Protein States. The Journal of Physical Chemistry B 119:5113-23. DOI: 10.1021/jp508971m.

Piana S., Klepeis JL., Shaw DE. 2014. Assessing the accuracy of physical models used in proteinfolding simulations: quantitative evidence from long molecular dynamics simulations. Current opinion in structural biology 24:98-105. DOI: 10.1016/j.sbi.2013.12.006.

Piana S., Lindorff-Larsen K., Shaw DE. 2011. How robust are protein folding simulations with respect to force field parameterization? Biophysical journal 100:L47-9. DOI: 10.1016/j.bpj.2011.03.051.

Piana S., Lindorff-Larsen K., Shaw DE. 2012. Protein folding kinetics and thermodynamics from atomistic simulation. Proceedings of the National Academy of Sciences of the United States of America 109:17845-50. DOI: 10.1073/pnas.1201811109.

Pitera JW., Chodera JD. 2012. On the Use of Experimental Observations to Bias Simulated Ensembles. Journal of Chemical Theory and Computation 8:3445-3451. DOI: $10.1021 / \operatorname{ct} 300112 \mathrm{v}$.

Pronk S., Páll S., Schulz R., Larsson P., Bjelkmar P., Apostolov R., Shirts MR., Smith JC., Kasson PM., van der Spoel D., Hess B., Lindahl E. 2013. GROMACS 4.5: a high-throughput and highly parallel open source molecular simulation toolkit. Bioinformatics 29:845-54. DOI: 10.1093/bioinformatics/btt055.

Qin BY., Liu C., Srinath H., Lam SS., Correia JJ., Derynck R., Lin K. 2005. Crystal structure of IRF-3 in complex with CBP. Structure 13:1269-77. DOI: 10.1016/j.str.2005.06.011.

Rauscher S., Gapsys V., Gajda MJ., Groot BL De., Grubmüller H. 2015. Structural Ensembles of Intrinsically Disordered Proteins Depend Strongly on Force Field: A Comparison to Experiment Structural Ensembles of Intrinsically Disordered Proteins Depend Strongly on Force Field: A Comparison to Experiment. Journal of Chemical Theory and Computation 11:5513-5524. DOI: 10.1021/acs.jctc.5b00736.

Ravera E., Sgheri L., Parigi G., Luchinat C. 2016. A critical assessment of methods to recover information from averaged data. Physical chemistry chemical physics : PCCP 18:5686-701. DOI: $10.1039 / \mathrm{c} 5 \mathrm{cp} 04077 \mathrm{a}$.

Robustelli P., Cavalli A., Dobson CM., Vendruscolo M., Salvatella X. 2009. Folding of small 
718

719

720

721

722

723

724

725

726

727

728

729

730

731

732

733

734

735

736

737

738

739

740

741

742

743

744

745

746

proteins by Monte Carlo simulations with chemical shift restraints without the use of molecular fragment replacement or structural homology. The journal of physical chemistry. B 113:7890-6. DOI: 10.1021/jp900780b.

Robustelli P., Kohlhoff K., Cavalli A., Vendruscolo M. 2010. Using NMR chemical shifts as structural restraints in molecular dynamics simulations of proteins. Structure 18:923-33. DOI: $10.1016 /$ j.str.2010.04.016.

Roux B., Weare J. 2013. On the statistical equivalence of restrained-ensemble simulations with the maximum entropy method. The Journal of chemical physics 138:84107. DOI: 10.1063/1.4792208.

Sahakyan AB., Vranken WF., Cavalli A., Vendruscolo M. 2011. Structure-based prediction of methyl chemical shifts in proteins. Journal of biomolecular NMR 50:331-46. DOI: 10.1007/s10858-011-9524-2.

Shaw DE., Maragakis P., Lindorff-Larsen K., Piana S., Dror RO., Eastwood MP., Bank JA., Jumper JM., Salmon JK., Shan Y., Wriggers W. 2010. Atomic-level characterization of the structural dynamics of proteins. Science 330:341-6. DOI: 10.1126/science.1187409.

Shen Y., Lange O., Delaglio F., Rossi P., Aramini JM., Liu G., Eletsky A., Wu Y., Singarapu KK., Lemak A., Ignatchenko A., Arrowsmith CH., Szyperski T., Montelione GT., Baker D., Bax A. 2008. Consistent blind protein structure generation from NMR chemical shift data. Proceedings of the National Academy of Sciences of the United States of America 105:468590. DOI: 10.1073/pnas.0800256105.

De Simone A., Aprile FA., Dhulesia A., Dobson CM., Vendruscolo M. 2015. Structure of a lowpopulation intermediate state in the release of an enzyme product. elife 4. DOI: 10.7554/eLife.02777.

De Simone A., Richter B., Salvatella X., Vendruscolo M. 2009. Toward an accurate determination of free energy landscapes in solution states of proteins. Journal of the American Chemical Society 131:3810-1. DOI: 10.1021/ja8087295.

Svergun D., Barberato C., Koch MHJ. 1995. CRYSOL - a Program to Evaluate X-ray Solution Scattering of Biological Macromolecules from Atomic Coordinates. Journal of Applied Crystallography 28:768-773. DOI: 10.1107/S0021889895007047. 
747 Tang C., Schwieters CD., Clore GM. 2007. Open-to-closed transition in apo maltose-binding 748 protein observed by paramagnetic NMR. Nature 449:1078-82. DOI: 10.1038/nature06232.

749 Theobald DL., Steindel PA. 2012. Optimal simultaneous superpositioning of multiple structures 750 with missing data. Bioinformatics 28:1972-1979. DOI: 10.1093/bioinformatics/bts243.

751 Tiberti M., Papaleo E., Bengtsen T., Boomsma W., Lindorff-Larsen K. 2015. ENCORE: Software 752 for quantitative ensemble comparison. PLoS computational biology 11:e1004415.

753 Tropp J. 1980. Dipolar relaxation and nuclear Overhauser effects in nonrigid molecules: The effect 754 755 of fluctuating internuclear distances. The Journal of Chemical Physics 72:6035. DOI: 10.1063/1.439059.

756

757

758

759

760

761

762

763

764 765

766

767 768

769

770

771

772

773

774

Vögeli B., Orts J., Strotz D., Chi C., Minges M., Wälti MA., Güntert P., Riek R. 2014. Towards a true protein movie: a perspective on the potential impact of the ensemble-based structure determination using exact NOEs. Journal of magnetic resonance 241:53-9. DOI: 10.1016/j.jmr.2013.11.016.

Waters L., Yue B., Veverka V., Renshaw P., Bramham J., Matsuda S., Frenkiel T., Kelly G., Muskett F., Carr M., Heery DM. 2006. Structural diversity in p160/CREB-binding protein coactivator complexes. The Journal of biological chemistry 281:14787-95. DOI: 10.1074/jbc.M600237200.

White AD., Voth GA. 2014. Efficient and Minimal Method to Bias Molecular Simulations with Experimental Data. Journal of Chemical Theory and Computation 10:3023-3030. DOI: $10.1021 / \operatorname{ct} 500320 \mathrm{c}$.

Wishart DS., Arndt D., Berjanskii M., Tang P., Zhou J., Lin G. 2008. CS23D: a web server for rapid protein structure generation using NMR chemical shifts and sequence data. Nucleic acids research 36:W496-502. DOI: 10.1093/nar/gkn305.

Wishart DS., Case DA. 2001. Use of chemical shifts in macromolecular structure determination. Methods in enzymology 338:3-34.

Zijlstra N., Dingfelder F., Wunderlich B., Zosel F., Benke S., Nettels D., Schuler B. 2017. Rapid Microfluidic Dilution for Single-Molecule Spectroscopy of Low-Affinity Biomolecular Complexes. Angewandte Chemie 129:7232-7235. DOI: 10.1002/ange.201702439. 


\section{Figure 1}

A previously determined structural model of the conformation of NCBD in solution

The structure is shown as a cartoon (PDB entry: $2 \mathrm{KKJ}$ ) with the protein coloured from the $\mathrm{N}$ to the C-terminal (blue to red). The three $\alpha$-helices are labelled. The goal of this work is to provide an ensemble of structures that represent the conformational fluctuations associated with this average conformation. 


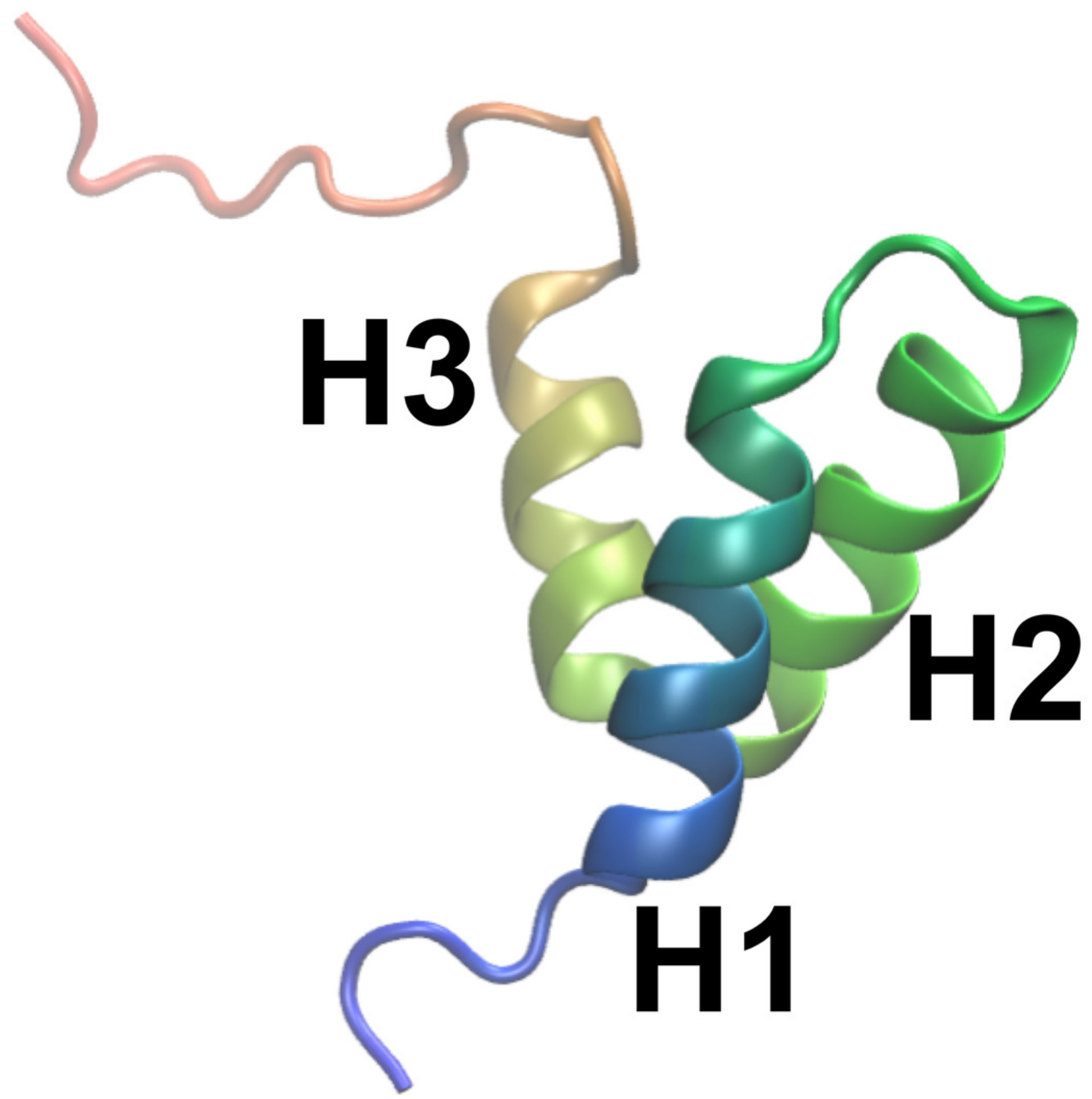




\section{Figure 2 (on next page)}

Assessment of the convergence of the simulations.

The similarity between structural ensembles was quantified using structural clustering with Affinity Propagation and subsequent comparison of the ensembles by Jensen-Shannon (JS) divergence. The JS divergence between two identical ensembles is zero, and it has previously been found that values less than $\sim 0.3$ represent similar ensembles. We monitored the evolution of the JS-divergence in two different tests, either by comparing two replicas from the same simulation (i.e. CS-NOE-2, black) or two simulations with the same force field and restraints but different starting structures (i.e. CS-NOE-2 starting from $2 \mathrm{KKJ}$ and 1 ZOQ structures, respectively, grey). As described in the text we discarded the first 45 SA cycles before calculating the ensemble similarity for the test with different starting structures. 


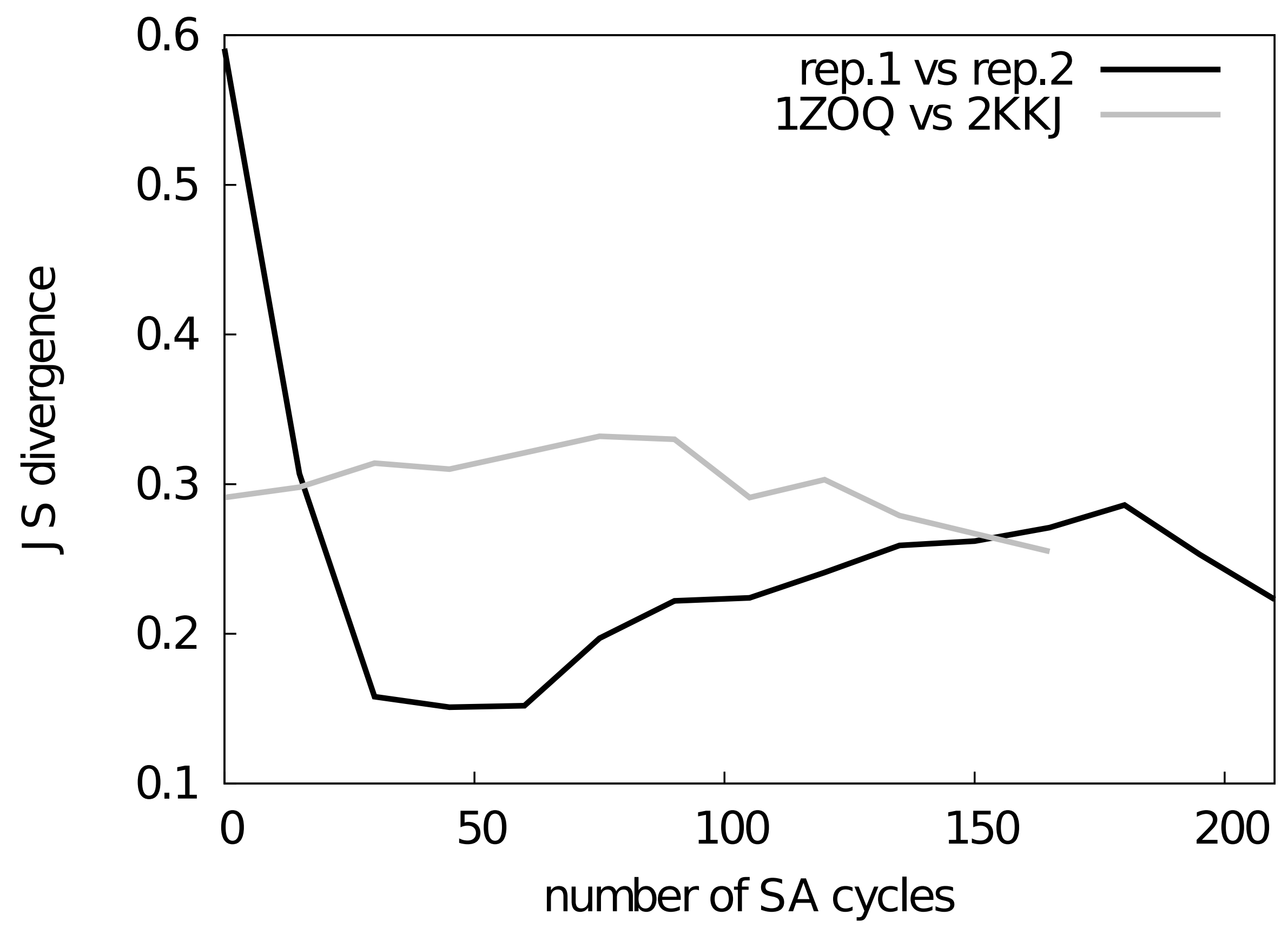




\section{Figure 3 (on next page)}

Validation of the structural ensemble using side-chain methyl chemical shifts.

We calculated the deviation between experimental and predicted $(A){ }^{13} \mathrm{C}$ and $(B){ }^{1} \mathrm{H}$ sidechain chemical shifts from each MD ensemble. The results are shown as a function of the number of replicas used for the averaging of the simulations. The previously determined NMR structure (black) and unbiased MD simulation (green) do not involve replica averaging and are shown as horizontal lines. 

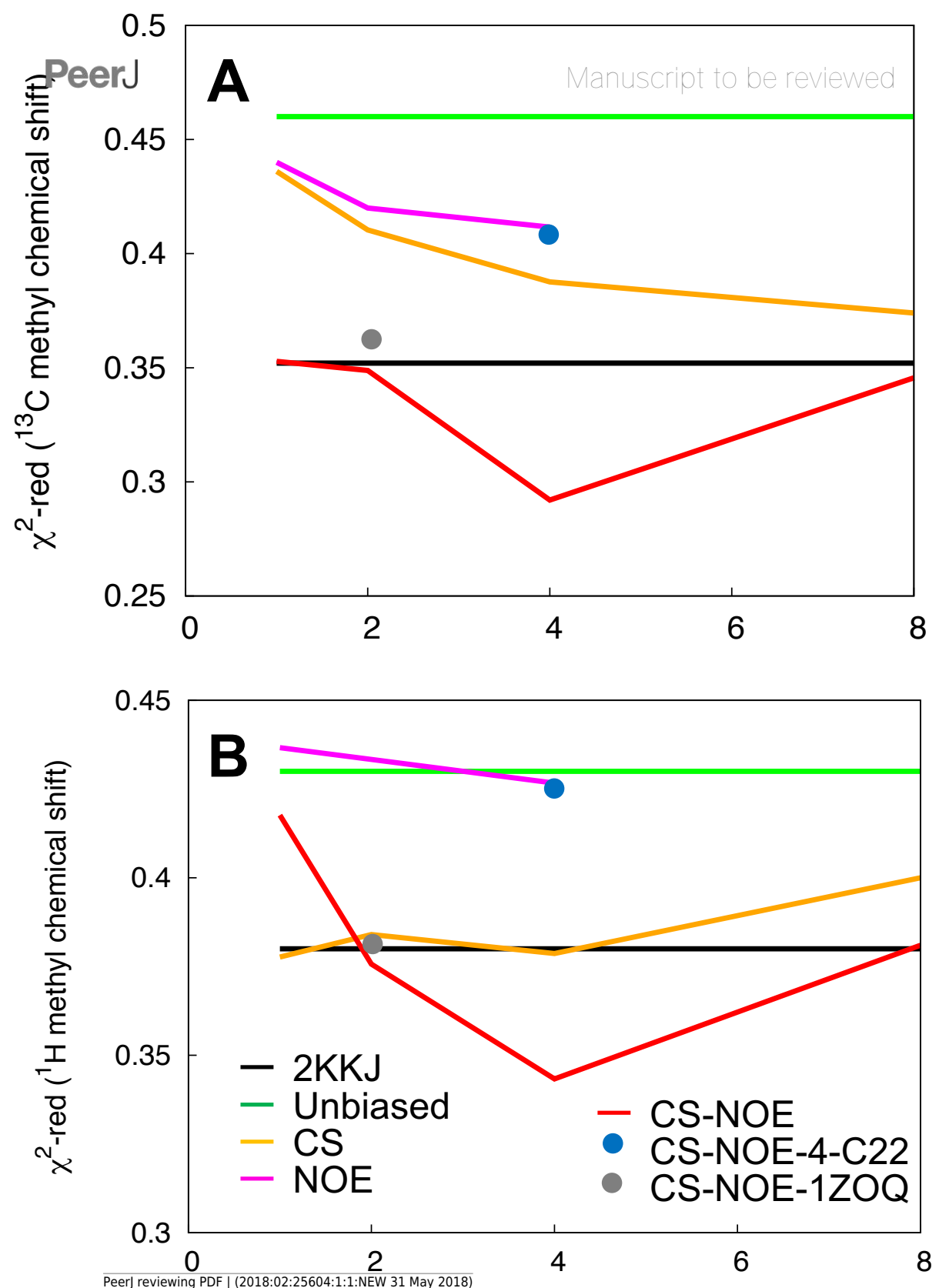

number of replicas 


\section{Figure 4}

Conformational ensemble of the free state of NCBD obtained by molecular dynamics simulations with the CHARMM22* force field and replica-averaged CS and NOE restraints.

The $\alpha$-helices are represented as cylinders and the structural ensemble was aligned using THESEUS. 


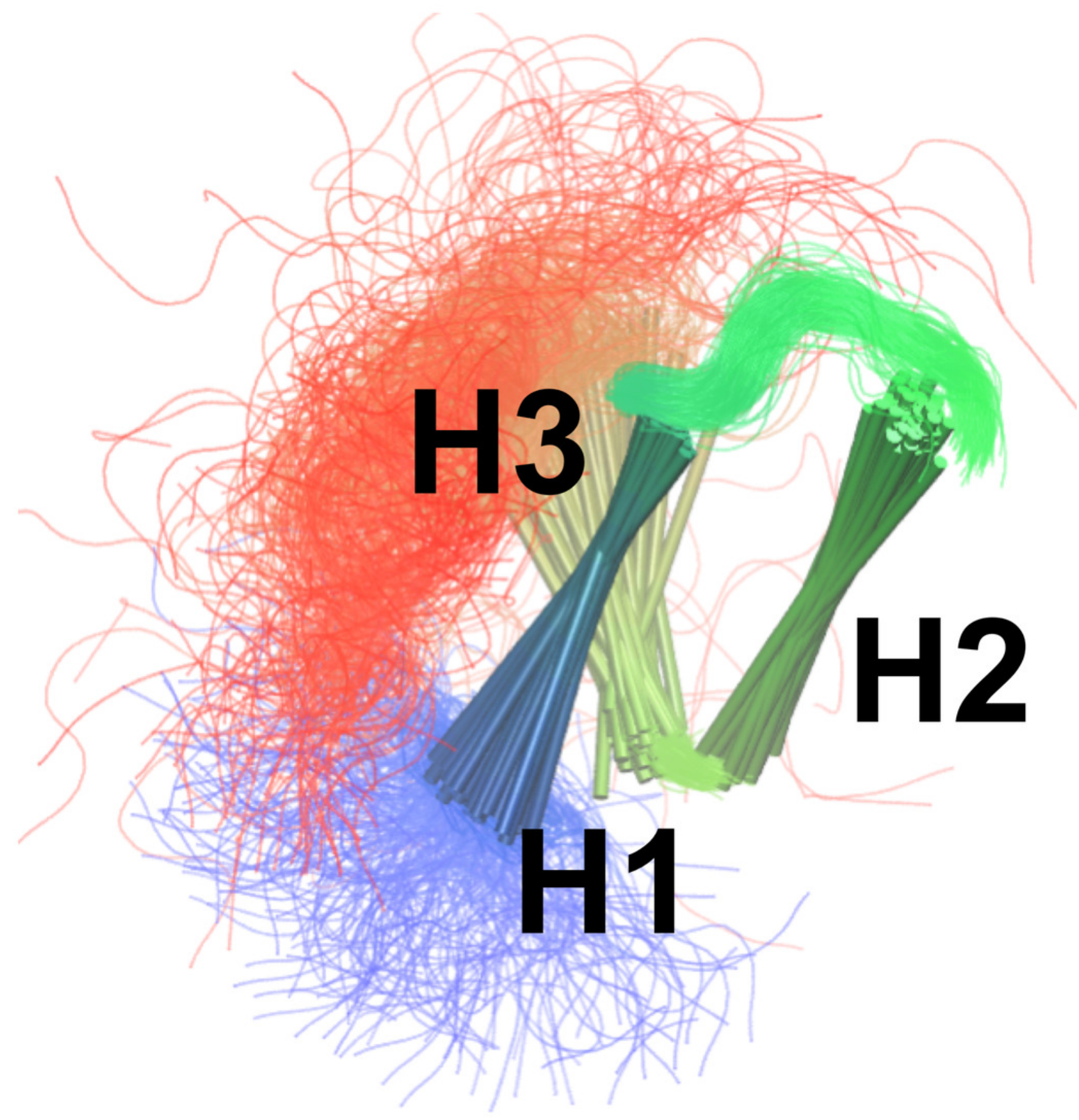


Figure $\mathbf{5}$ (on next page)

Calculation of order parameters from MD simulations to probe short and long timescale dynamics.

We calculated $S^{2}$ order parameters that reflect either motions faster than overall tumbling of the protein (black) or longer timescale motions that give rise to chemical shift and NOE averaging (red). For reference, the main chain Root Mean Square Deviation (RMSD) values of the 28 unbiased simulations that we used to calculate the $S_{\text {relaxation }}^{2}$ values are shown in Figure S3. 


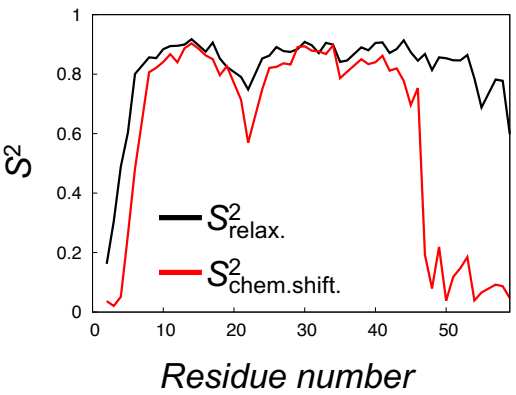

